\title{
OPEN Wetting Properties of Graphene Aerogels
}

\author{
Francesco De Nicola ${ }^{1 *}$, Ilenia Viola ${ }^{2}$, Lorenzo Donato Tenuzzo ${ }^{3}$, Florian Rasch ${ }^{4}$, \\ Martin R. Lohe ${ }^{5}$, Ali Shaygan $\mathrm{Nia}^{5}$, Fabian Schütt $\mathbb{D}^{4}$, Xinliang Feng $\mathbb{D}^{5}$, Rainer Adelung ${ }^{4}$ \& \\ Stefano Lupi ${ }^{1,3}$
}

Graphene hydrophobic coatings paved the way towards a new generation of optoelectronic and fluidic devices. Nevertheless, such hydrophobic thin films rely only on graphene non-polar surface, rather than taking advantage of its surface roughness. Furthermore, graphene is typically not selfstanding. Differently, carbon aerogels have high porosity, large effective surface area due to their surface roughness, and very low mass density, which make them a promising candidate as a superhydrophobic material for novel technological applications. However, despite a few works reporting the general super-hydrophobic and lipophilic behavior of the carbon aerogels, a detailed characterization of their wetting properties is still missing, to date. Here, the wetting properties of graphene aerogels are demonstrated in detail. Without any chemical functionalization or patterning of their surface, the samples exhibit a super-lipophilic state and a stationary super-hydrophobic state with a contact angle up to $150 \pm 15^{\circ}$ and low contact angle hysteresis $\approx 15^{\circ}$, owing to the fakir effect. In addition, the adhesion force of the graphene aerogels in contact with the water droplets and their surface tension are evaluated. For instance, the unique wettability and enhanced liquid absorption of the graphene aerogels can be exploited for reducing contamination from oil spills and chemical leakage accidents.

In general, the realization of artificial hydrophobic surfaces depends on the material surface chemical composition and its morphological structure. Although the chemical composition is an intrinsic material property, it can be engineered to decrease the solid surface tension ${ }^{1}$, therefore increase the hydrophobicity of the surface. On the other hand, surface roughness ${ }^{2,3}$ (micro- and nano-morphology) may also enhance the hydrophobicity, particularly by exploiting hierarchical ${ }^{2,4-7}$ and fractal architectures ${ }^{7,8}$, that allow the formation of air pockets to prevent water imbibition. Nonetheless, the fabrication of a permanent super-hydrophobic surface is a challenging task. Recently, time durability ${ }^{5-7}$, chemical $^{9}$, mechanical ${ }^{10}$, and thermal stability ${ }^{11}$ have been addressed. Among the numerous materials having the two aforementioned features, graphene offers versatility, stability, and multi-functionality owing to its unique optical ${ }^{12}$ and electronic ${ }^{13}$ properties, making its usage widespread in hydrophobic surface realizations ${ }^{14-16}$.

Graphene is constituted by a $s p^{2}$ lattice of graphitic carbon, thus it has a slightly hydrophilic (graphite contact angle $\approx 86^{\circ 17}$ ) but non-polar surface. Nevertheless, surface functionalization and substrate interactions may be exploited to tailor graphene wetting properties in a controlled fashion ${ }^{15,16}$. However, graphene has been used so far to realize hydrophobic coatings only employing its non-polar surface, rather than taking advantage of its surface roughness. Furthermore, graphene is not self-standing in most applications, acting thus as a coating.

On the other hand, carbon aerogels ${ }^{18-22}$ are carbon-based, macroscopic, three-dimensional structures characterized by a randomly crosslinking network of hierarchical nanostructures and microstructures. Typically, aerogels have high porosity, very low mass density, and large effective surface area due to their hierarchical high surface roughness, which make them a promising candidate as a super-hydrophobic material for novel technological applications. Despite a few works ${ }^{19-22}$ reporting the observation of a super-hydrophobic and lipophilic behavior in carbon aerogels, the characterization of the wetting properties of such a class of materials is still missing, to date.

\footnotetext{
${ }^{1}$ Graphene Labs, Istituto Italiano di Tecnologia, Via Morego 30, 16163, Genova, Italy. ${ }^{2}$ CNR NANOTEC-Institute of Nanotechnology, S.Li.M. Lab, Department of Physics, University of Rome La Sapienza, P.le A. Moro 5, 00185, Roma, Italy. ${ }^{3}$ Department of Physics, University of Rome La Sapienza, P.le A. Moro 5, 00185, Roma, Italy. ${ }^{4}$ Functional Nanomaterials, Institute for Materials Science, Kiel University, Kaiser Str. 2, 24143, Kiel, Germany. ${ }^{5}$ Center for Advancing Electronics Dresden (CFAED) \& Department of Chemistry and Food Chemistry, Technische Universität Dresden, Helmholtzstraße 10, 01069, Dresden, Germany.*email: francesco.denicola@iit.it
} 


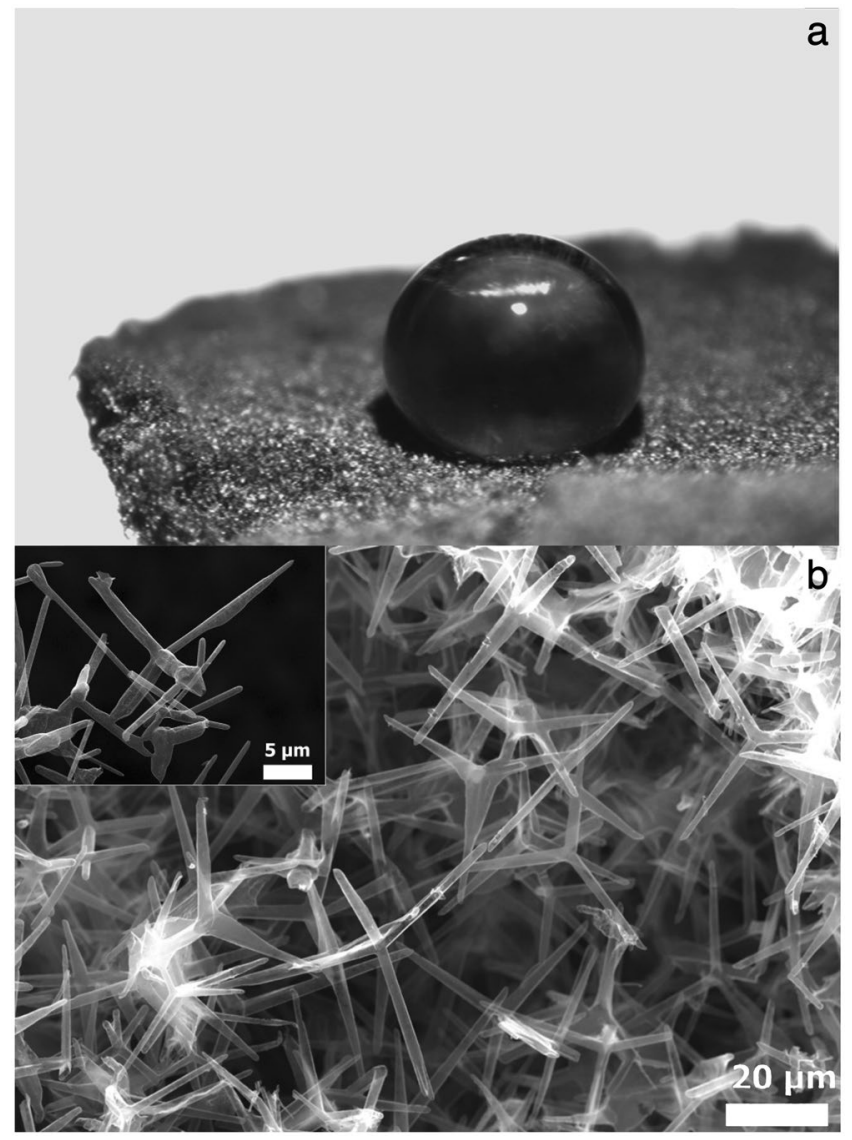

Figure 1. Aerographene aerogels morphology. (a), Image of a water droplet cast on a hydrophobic Aerographene aerogel. (b), Representative SEM micrograph of the Aerographene aerogel surface. Inset, Detail of carbon tetrapods.

Here, we give insight on the wetting properties of graphene aerogels in detail. In particular, we show that owing to their particular morphology and high surface porosity (up to 0.81 ), our graphene aerogels exhibit a super-lipophilic state and a stationary super-hydrophobic state, achieving high apparent contact angle values $\theta^{\circ} \geq 150^{\circ}$, with low contact angle hysteresis ${ }^{1}(\mathrm{CAH}) \approx 15^{\circ}$, and high work of adhesion $\left(10 \mathrm{~mJ} / \mathrm{m}^{2}\right)$. In addition, we characterized the graphene aerogel solid surface tension $(63 \mathrm{mN} / \mathrm{m})$.

\section{Results and Discussion}

The graphene aerogels studied are made of a cellular material called Aerographene (see Methods). The samples have a square-shaped surface with a lateral size $L=2 \mathrm{~cm}$, a thickness $d=0.2 \mathrm{~cm}$, and a mass $m=5-15$ mg. A representative sample is reported in Fig. 1a. From the scanning electron microscopy (SEM) micrograph in Fig. 1b, a random network made of micrometer-sized carbon tetrapods ${ }^{23-25}$ constituting the aerogel surface (Fig. 1b, inset) can be observed. This particular shape is due to the precursor $\mathrm{ZnO}$ template employed during the material synthesis. Therefore, such a highly porous (volume porosity up to $\approx 0.99$ ) system cannot be considered as an homogeneous solid.

The mass density of the Aerographene aerogels can be estimated at first approximation by $\rho=m / V$. However, this is the total mass density, as aerogels are matter with a mixed phase of gas and solid. Hence, a better estimation of the solid mass density can be provided by the effective medium approximation $\rho=\rho_{c}\left(1-\Phi_{-}\right)+\rho_{\text {air }} \Phi_{-}$, with $\Phi_{c}+\Phi_{-}=1$, where $\rho_{c}=2200 \mathrm{~kg} / \mathrm{m}^{3}$ and $\Phi_{c}=V_{c} / V$ are respectively the density and the volume fraction of the carbon phase, while $\rho_{\text {air }}=1.225 \mathrm{~kg} / \mathrm{m}^{3}$ and $\Phi_{-}=V_{\text {air }} / V$ are the density and the volume fraction of air, respectively. Hence, the effective mass density is defined as $\rho_{e f f}=\rho_{c}\left(1-\Phi_{-}\right)$. Therefore, the aerogel porous fraction is $\Phi_{-}=0.992-0.998$, leading to an effective mass density down to $\rho_{\text {eff }}=5 \mathrm{~kg} / \mathrm{m}^{3}$.

Owing to their random surface morphology, the static contact angle on porous media can be defined only on average ${ }^{1}$. The contact angle of a water droplet cast on a representative Aerographene aerogel is shown in Fig. 2a. We measured a maximum apparent contact angle $\theta^{*}=150 \pm 15^{\circ}$ on our samples, thus the sample is super-hydrophobic $\left(\theta^{*} \geq 150^{\circ}\right)$. The error on the contact angle was evaluated by the $\mathrm{CAH}$, that is defined as the difference between the advancing and receding contact angle (Fig. 2b). Such a low CAH is due to the highly rough and porous surface of the samples and it is peculiar of the fakir effect ${ }^{26}$.

Moreover, we evaluated the work of adhesion of the aerogel surface in contact with water by the Young-Dupré equation ${ }^{1}$ 


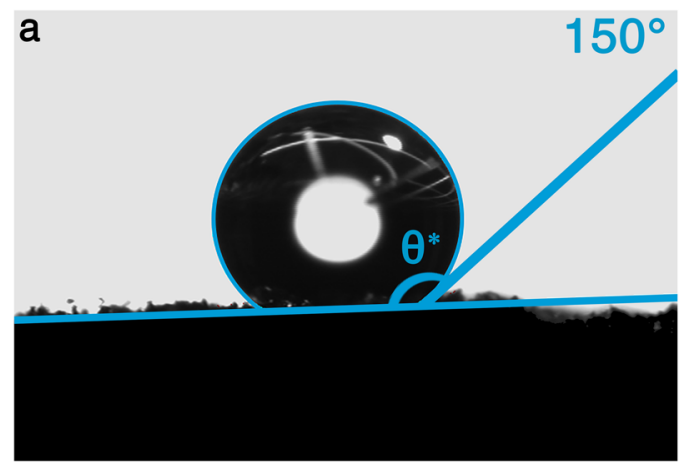

C

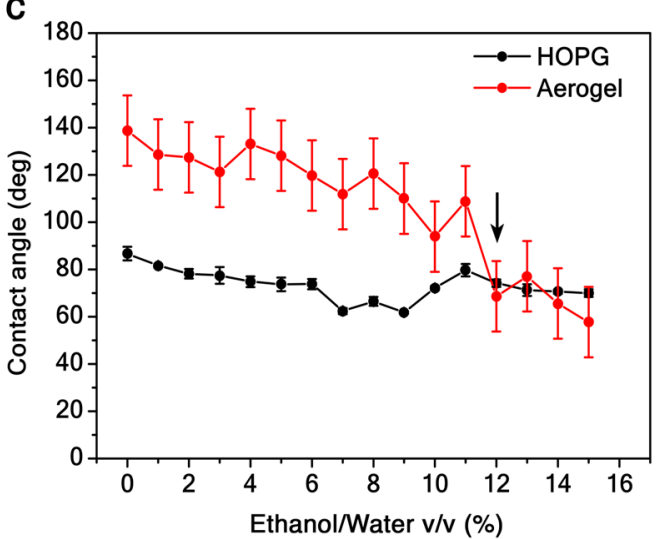

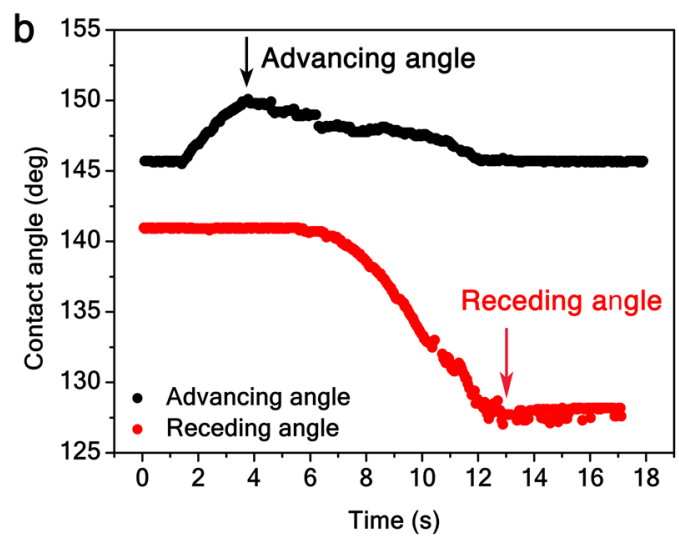

d

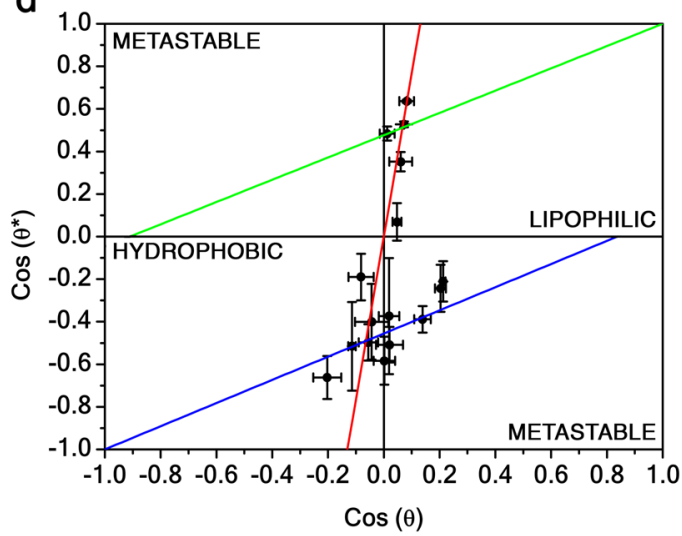

Figure 2. Contact angle measurements. (a), Image of a sessile water droplet cast on a super-hydrophobic $\left(\theta^{*}=150 \pm 15^{\circ}\right)$ Aerographene aerogel. (b), Contact angle as a function of time by increasing (black dots) and decreasing (red dots) the water droplet volume cast on the aerogel. The advancing and receding contact angles are marked. (c), Contact angles of Aerographene aerogel (red dots) and HOPG (black dots) as a function of the ethanol volume concentration in water. The black arrow marks the lipophilic transition point between the Wenzel and Cassie-Baxter regime. (d), Wenzel-Cassie-Baxter wetting phase diagram of the Aerographene aerogel surface with respect to the HOPG surface. Wetting states are studied changing the liquid surface tension by adding different concentrations in volume of ethanol in water. The fit of Wenzel equation (red solid line) reports a roughness factor $r=7.6 \pm 0.4$, while the fits of lipophilic (green solid line) and hydrophobic (blue solid line) Cassie-Baxter equations report respectively a liquid fraction $\Phi_{+}=0.48 \pm 0.03$ and an air fraction $\Phi_{-}=0.45 \pm 0.02$. The Wenzel-Cassie-Baxter transition point in the hydrophobic regime is the intersection between the red and blue solid lines, while in the lipophilic regime it is the intersection between the red and green solid lines. Error bars are standard deviations of data measured over different sample areas.

$$
W_{a d h}=\gamma_{L V}\left(1+\cos \theta^{*}\right)
$$

For the super-hydrophobic sample in Fig. $2 \mathrm{a}, W_{a d h} \approx 10 \mathrm{~mJ} / \mathrm{m}^{2}$. Therefore, for a water drop with $1 \mathrm{~mm}$ diameter, the adhesion force of the Aerographene aerogel in contact with the drop is $F_{a d h} \approx 10 \mu \mathrm{N}$. For instance, the obtained result is about $17 \%$ lower than the adhesion force of a single gecko foot-hair ${ }^{27}$, but 10 times higher than that of Salvinia leaf ${ }^{28}$, and compatible with the value reported for carbon nanotube films ${ }^{6}$.

In order to better understand the wettability of Aerographene aerogels, we characterized their wetting states with respect to a highly oriented pyrolytic graphite (HOPG) reference substrate (Bruker). In Fig. 2c, we report the contact angle of the super-hydrophobic aerogel and the HOPG as a function of the concentration in volume of ethanol in water. Since ethanol has a lower liquid-vapor surface tension $\left(\gamma_{L V}=22 \mathrm{mN} / \mathrm{m}\right)$ than water $\left(\gamma_{L V}=72\right.$ $\mathrm{mN} / \mathrm{m}$ ), the higher the ethanol concentration, the lower the surface tension of the overall solution.

Generally, the contact angle between a chemically homogeneous solid surface and a liquid droplet obeys to the Young relation ${ }^{1}$

$$
\cos \theta=\frac{\gamma_{S V}-\gamma_{S L}}{\gamma_{L V}}
$$

where $\gamma_{S V}$ and $\gamma_{S L}$ are the solid-vapor and solid-liquid surface tensions, respectively. Therefore, the lower the surface tension of the liquid droplet, the lower the Young contact angle of the HOPG. This phenomenon is connected to the lipophilicity of the non-polar surface of carbon-based materials. Indeed, a minimum contact angle 
$\left(\theta=70^{\circ}\right)$ can be measured on the HOPG for pure ethanol droplets (Fig. 2c). On the other hand, it was not possible to measure the aerogel contact angle with acetone, ethanol, and glycerol because the material instantly and completely absorbed the droplets. Hence, the Aerographene aerogels are super-lipophilic $\left(\theta^{*}<5^{\circ}\right)$ as a consequence of their super-hydrophobic behavior. We further observed that for $\theta=77^{\circ},\left(\cos \theta^{*}=0.2\right)$ there is an intersection point between the two curves in Fig. 2c, beyond which the aerogel surface becomes more lipophilic than the graphite surface. That point corresponds to the transition point from the Wenzel to the Cassie-Baxter state in the lipophilic regime of the Wenzel-Cassie-Baxter phase diagram ${ }^{5}$, as confirmed from the plot (first quadrant) in Fig. 2d. We fit our data with the lipophilic Cassie-Baxter equation ${ }^{7,29}$

$$
\cos \theta^{*}=\left(1-\phi_{+}\right) \cos \theta+\phi_{+}, \quad 1=\phi+\phi_{+},
$$

by considering the Aerographene aerogel as a composite surface with a solid surface fraction $\Phi$, a surface fraction wetted by the liquid $\Phi_{+}$, an aerogel contact angle $\theta^{*}$, and a HOPG Young contact angle $\theta$. We obtained from the fit (green solid line) a liquid fraction $\Phi_{+}=0.48 \pm 0.03$ in contact with the droplet. We point out that metastable ${ }^{30}$ Cassie-Baxter states coexist with Wenzel states, which are stable as they are lower in surface free energy ${ }^{5}$. Therefore, we fit our data in Fig. 2d with the Wenzel equation ${ }^{3}$

$$
\cos \theta^{*}=r \cos \theta, \quad r \geq 1,
$$

where $r$ is the roughness factor (i.e., the ratio between the actual wet surface area and its geometrical projection on the plane) $)^{5}$. The fit (red solid line) returned $r=7.6 \pm 0.4$, meaning that the Aerographene aerogel surface is quite rough (the root-mean-squared roughness is $\approx 3 \mu \mathrm{m}$ ). In addition, from Eqs. 3 and 4 we obtain the relation

$$
\cos \theta=\frac{\phi_{+}}{r+\phi_{+}-1}
$$

from which we infer that the lipophilic Wenzel/Cassie-Baxter transition point occurs at $\cos \theta=0.07$ (the intersection between the red and green solid lines in Fig. 2d), hence confirming that the achieved lipophilic Cassie-Baxter states are metastable.

On the other hand, in the hydrophobic regime (third quadrant of the plot) we observe a continuous transition between the Wenzel and the Cassie-Baxter states beyond $\cos \theta=0$. Also, the plot depicts that the transition occurs by passing through metastable states extending into the fourth quadrant, slowing down the dewetting process. By fitting our data (blue solid line) in Fig. $2 \mathrm{~d}$ with the hydrophobic Cassie-Baxter equation ${ }^{7,29}$

$$
\cos \theta^{*}=\left(1-\phi_{-}\right) \cos \theta-\phi_{-}, \quad 1=\phi+\phi_{-},
$$

we obtained an air surface fraction $\Phi_{-}=0.45 \pm 0.02$ below the liquid droplet. Furthermore, from Eqs. 4 and 6 we obtain the relation

$$
\cos \theta=\frac{\phi_{-}}{1-r-\phi_{-}}
$$

from which we infer that the hydrophobic Wenzel/Cassie-Baxter transition point is at $\cos \theta=-0.07$ (the intersection between the red and blue solid lines in Fig. 2d), thus the maximum hydrophobic Cassie-Baxter state achieved is not metastable. These results suggest an air pocket formation. Therefore, we assert that the reason of the improved hydrophobicity/lipophilicity of the Aerographene aerogel over HOPG, is the fakir effect (high contact angle and low $\mathrm{CAH}$ ) induced by the aerogel microstructure. When the interaction of the surface with the liquid is hydrophobic, the particular surface morphology promotes the air pocket formation. Otherwise, when the interaction between the surface and the liquid is lipophilic, the morphology induces the development of a precursor liquid film ${ }^{1}$, improving the wetting behavior of the Aerographene aerogel surface. Furthermore, in composite rough surfaces, their morphology may favor a wetting transition from a Wenzel to a Cassie-Baxter state, due to air trapping ${ }^{5}$. This transition typically occurs by thermodynamically metastable states ${ }^{30}$.

In addition, we investigated the stability of the Aerographene aerogel hydrophobicity over time. Figure 3 reports the variations of the contact angle value as a function of the elapsed time since water was cast on the aerogel. In such an experiment, we show that despite the samples are porous, the contact angle is constant up to $10 \mathrm{~min}$. The slight linear decrease of the droplet radius in time is only due to the liquid evaporation and not to the suction by the aerogel, otherwise the contact angle would not be constant in time along with droplet radius ${ }^{1,7}$. In addition, we demonstrated a remarkable stability over several hours of the hydrophobic Cassie-Baxter state for the Aerographene aerogel (Supplementary Material). This result is particularly relevant, as the water contact angle of other carbon-based surfaces has been reported to decrease linearly with time, from an initial value of $146^{\circ}$ to 0 within $15 \mathrm{~min}^{31}$.

Moreover, we estimated the solid surface tension $\gamma_{S V}$ of the HOPG substrate (Fig. 4a) and the graphene aerogel (Fig. 4b) by the Neumann equation ${ }^{17}$

$$
\log \left[\gamma_{L V}\left(\frac{1+\cos \theta^{*}}{2}\right)^{2}\right]=\log \left(\gamma_{S V}\right)-\beta\left(\gamma_{L V}-\gamma_{S V}\right)^{2},
$$

where $\gamma_{L V}$ is the liquid-vapor surface tension of the different concentrations in volume of ethanol in water and $\beta$ is a constant. The fit in the lipophilic regime returns $\gamma_{S V} \approx 42 \mathrm{mN} / \mathrm{m}$ and $\gamma_{S V} \approx 63 \mathrm{mN} / \mathrm{m}$ for the HOPG and the graphene aerogel, respectively. 


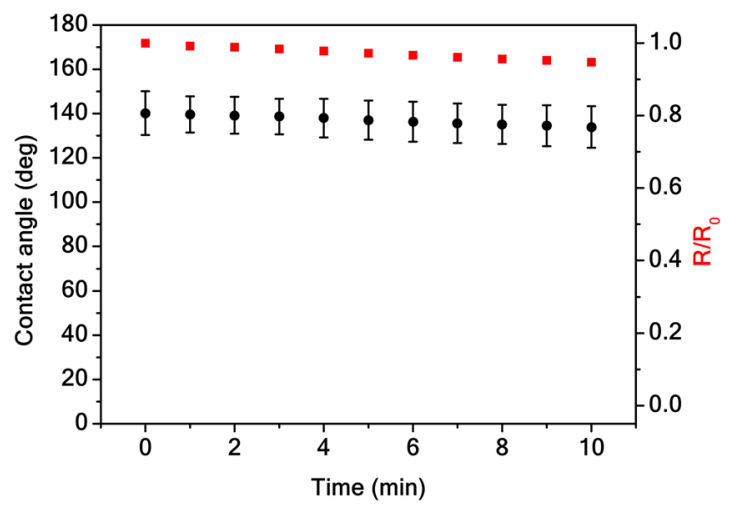

Figure 3. Wetting stability. Stability of the contact angle in the super-hydrophobic regime over time (black dots). Normalized radius of the water droplet as a function of the elapsed time (red squares). $R_{0}$ is the radius initial value.
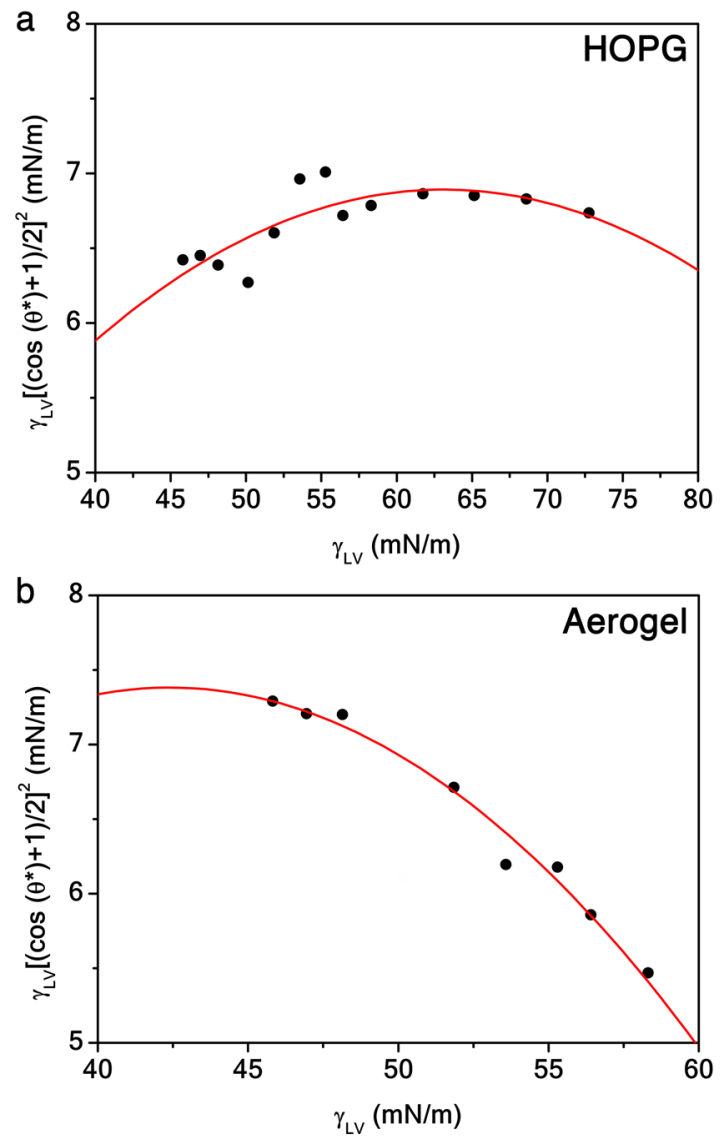

Figure 4. Solid surface tension characterization. Neumann equation in the lipophilic regime as a function of the liquid-vapor surface tension of different concentrations in volume of ethanol in water for the HOPG substrate (a) and the graphene aerogel (b). Red solid curves are quadratic polynomial fits.

In summary, we investigated the wetting properties of graphene aerogels. We demonstrated that such a material can be in a super-lipophilic, thus super-hydrophobic state that is stationary. The evaluated adhesion force of the water droplets with the aerogel is higher than the leaves of aquatic plants and we estimated the graphene aerogel solid surface tension. Stationary super-hydrophobic behavior of solid surfaces is a relevant property in a number of natural ${ }^{2}$ and technological processes ${ }^{32}$ with several industrial applications such as waterproof surfaces $^{33}$, anti-sticking ${ }^{34}$, anti-contamination ${ }^{4}$, self-cleaning ${ }^{32}$, anti-fouling ${ }^{35}$, anti-fogging ${ }^{36}$, low-friction coatings ${ }^{10}$, adsorption $^{37}$, lubrication ${ }^{17}$, dispersion ${ }^{1}$, self-assembly ${ }^{5}$, and optoelectronic and fluidic devices ${ }^{38,39}$. 


\begin{abstract}
Methods
Fabrication of the aerographene aerogels. A macroscopic $(2 \times 2 \times 0.5 \mathrm{~cm})$ and highly porous $(\approx 0.94)$ template consisting of interconnected ceramic $\mathrm{ZnO}$ tetrapods $\mathrm{s}^{23-25}$ was infiltrated with an aqueous dispersion $(2$ $\mathrm{mg} / \mathrm{ml}$ ) of electrochemically exfoliated graphene flakes ${ }^{40}$. After evaporation of the solvent and self-assembly of the graphene flakes on the template surface, the $\mathrm{ZnO}$ template was removed by chemical etching in $1 \mathrm{M} \mathrm{HCl}$ and subsequent critical point drying resulted in a macroscopic, free-standing random network of hollow, interconnected carbon tetrapods having a wall thickness on the order of a few nanometers, length $\approx 20 \mu \mathrm{m}$, and diameter about a few micrometers.
\end{abstract}

Contact angle measurements. Images of sessile water drops deposited on Aerographene aerogels were acquired by Dataphysics OCA instrument and analyzed by its software. In order to estimate the average, maximum, and minimum contact angle on the aerogel surface, static, advancing, and receding contact angles ${ }^{1}$ were measured, respectively, by increasing and decreasing the volume of the drop by $1 \mu \mathrm{L}$ steps. In static contact angle measurements, the volume of the deionized $(18.2 \mathrm{M} \Omega \mathrm{cm})$ water droplet was $V=15 \mu \mathrm{L}$. Every contact angle was measured $15 \mathrm{~s}$ after drop casting to ensure that the droplet had reached its equilibrium position, and it was averaged over the values obtained in different areas of the sample surface. Due to the heterogeneous and porous aerogel surface, we evaluated the error on the contact angle values by $C A H \approx 15^{\circ}$. The experimental contact angle probably corresponds to a lower limit, as the water droplet might cause some small dimple on the aerogel surface. The calculated surface air and liquid fractions are effective values, as they were determined by macroscopic techniques relying on the surface properties of the material. The liquid-vapor surface tension of the concentrations in volume of ethanol in water was measured by the pendant drop method ${ }^{1}$.

Received: 10 July 2019; Accepted: 22 January 2020;

Published online: 05 February 2020

\title{
References
}

1. De Gennes, P.-G., Brochard-Wyart, F. \& Quéré, D.Capillarity and wetting phenomena (Springer, New York, 2003)

2. Feng, L. et al. Petal effect: A superhydrophobic state with high adhesive force. Langmuir 24, 4114-4119 (2008).

3. Wenzel, R. N. Resistance of solid surfaces to wetting by water. Ind. Eng. Chem 28, 988-994 (1936).

4. Li, Y. et al. Superhydrophobic bionic surfaces with hierarchical microsphere/swcnt composite arrays. Langmuir 23, 2169-2174 (2007).

5. De Nicola, F. et al. Exploiting the hierarchical morphology of single-walled and multi-walled carbon nanotube films for highly hydrophobic coatings. Beilstein J. Nanotechnol 6, 353-360 (2015).

6. De Nicola, F. et al. Super-hydrophobic multi-walled carbon nanotube coatings for stainless steel. Nanotechnology 26, 1457011-1457016 (2015).

7. De Nicola, F. et al. Multi-fractal hierarchy of single-walled carbon nanotube hydrophobic coatings. Sci. Rep. 5, 1-9 (2015).

8. Shibuichi, S., Onda, T., Satoh, N. \& Tsujii, K. Super water-repellent surfaces resulting from fractal structure. J. Phys. Chem. 100, 19512-19517 (1996).

9. Wang, K., Hu, N.-X., Xu, G. \& Qi, Y. Stable superhydrophobic composite coatings made from an aqueous dispersion of carbon nanotubes and a fluoropolymer. Carbon 49, 1769-1774 (2011).

10. Jung, Y. C. \& Bhushan, B. Mechanically durable carbon nanotube-composite hierarchical structures with superhydrophobicity, selfcleaning, and low-drag. ACS Nano 3, 4155-4163 (2009).

11. Wang, N. et al. Long-term and thermally stable superhydrophobic surfaces of carbon nanofibers. J. Colloid Interface Sci. 320, 365-368 (2008)

12. Grigorenko, A. N., Polini, M. \& Novoselov, K. S. Graphene plasmonics. Nat. Photonics 6, 749-758 (2012).

13. Banszerus, L. et al. Ultrahigh-mobility graphene devices from chemical vapor deposition on reusable copper. Sci. Adv 1, 1-6 (2015).

14. Wang, S., Zhang, Y., Abidi, N. \& Cabrales, L. Wettability and surface free energy of graphene films. Langmuir 25, 11078-11081 (2009).

15. Rafiee, J., Rafiee, M. A., Yu, Z.-Z. \& Koratkar, N. Superhydrophobic to superhydrophilic wetting control in graphene films. Adv. Mater. 22, 2151-2154 (2010).

16. Shin, Y. J. et al. Surface-energy engineering of graphene. Langmuir 26, 3798-3802 (2010).

17. Adamson, A. W. \& Gast, A. P. Physical Chemistry of Surfaces (John Wiley \& Sons, New York, 1997)

18. Mecklenburg, M. et al. Aerographite: Ultra lightweight, flexible nanowall, carbon microtube material with outstanding mechanical performance. Adv. Mater. 24, 3486-3490 (2012).

19. Lin, Y., Ehlert, G. J., Bukowsky, C. \& Sodano, H. A. Superhydrophobic functionalized graphene aerogels. ACS Appl. Mater. Interfaces 3,2200-2203 (2011).

20. Wu, Z.-Y. et al. Carbon nanofiber aerogels for emergent cleanup of oil spillage and chemical leakage under harsh conditions. Sci. Rep. 4, 1-6 (2014).

21. Luo, Y., Jiang, S., Xiao, Q., Chen, C. \& Li, B. Highly reusable and superhydrophobic spongy graphene aerogels for efficient oil/water separation. Sci. Rep. 7, 1-10 (2017).

22. Scarselli, M. et al. Applications of three-dimensional carbon nanotube networks. Beilstein J. Nanotechnol 6, 792-798 (2015).

23. Mishra, Y. K. et al. Fabrication of macroscopically flexible and highly porous $3 \mathrm{D}$ semiconductor networks from interpenetrating nanostructures by a simple flame transport approach. Part. Part. Syst. Charact. 30, 775-783 (2013).

24. Meija, R. et al. Nanomechanics of individual aerographite tetrapods. Nat. Commun. 8, 1-9 (2017).

25. Taale, M. et al. Biomimetic carbon fiber systems engineering: A modular design strategy to generate biofunctional composites from graphene and carbon nanofibers. ACS Appl. Mater. Interfaces 11, 5325-5335 (2019).

26. Quéré, D. Fakir droplets. Nat. Mater. 1, 14-15 (2002).

27. Autumn, K. et al. Adhesive force of a single gecko foot-hair. Nature 405, 681-685 (2000).

28. Hunt, J. \& Bhushan, B. Nanoscale biomimetics studies of salvinia molesta for micropattern fabrication. J. Colloid Interface Sci. 363, 187-192 (2011).

29. Cassie, A. B. D. \& Baxter, S. Wettability of porous surfaces. J. Chem. Soc. Faraday Trans. 40, 546-551 (1944).

30. Giacomello, A., Chinappi, M., Meloni, S. \& Casciola, C. M. Metastable wetting on superhydrophobic surfaces: Continuum and atomistic views of the Cassie-Baxter-Wenzel transition. Phys. Rev. Lett. 109, 226102 (2012).

31. Huang, L., Lau, S. P., Yang, H. Y., Leong, E. S. P. \& Yu, S. F. Stable superhydrophobic surface via carbon nanotubes coated with a zno thin film. J. Phys. Chem. B 109, 7746-7748 (2005). 
32. Fürstner, R., Barthlott, W., Neinhuis, C. \& Walzel, P. Wetting and self-cleaning properties of artificial superhydrophobic surfaces. Langmuir 21, 956-961 (2005).

33. Lau, K. K. S. et al. Superhydrophobic carbon nanotube forests. Nano Lett. 3, 1701-1705 (2003).

34. Wang, Z., Koratkar, N., Ci, L. \& Ajayan, P. M. Combined micro-/nanoscale surface roughness for enhanced hydrophobic stability in carbon nanotube arrays. Appl. Phys. Lett. 90, 143117 (2007).

35. Zhang, H., Lamb, R. \& Lewis, J. Engineering nanoscale roughness on hydrophobic surface-preliminary assessment of fouling behaviour. Sci. Technol. Adv. Mater. 6, 236-239 (2005).

36. Lai, Y. et al. Transparent superhydrophobic/superhydrophilic $\mathrm{TiO}_{2}$-based coatings for self-cleaning and anti-fogging. J. Mater. Chem. 22, 7420 (2012).

37. Li, J., Wang, L. \& Jiang, W. Super-hydrophobic surface of bulk carbon nanotubes compacted by spark plasma sintering followed by modification with polytetrofluorethylene. Carbon 48, 2644-2673 (2010).

38. Fang, C. et al. Tunable optical limiting optofluidic device filled with graphene oxide dispersion in ethanol. Sci. Rep. 5, 1-10 (2015).

39. Min, S. K., Kim, W. Y., Cho, Y. \& Kim, K. S. Fast dna sequencing with a graphene-based nanochannel device. Nat. Nanotechnol 6, 162-165(2011).

40. Parvez, K. et al. Exfoliation of graphite into graphene in aqueous solutions of inorganic salts. J. Am. Chem. Soc. 136, 6083-6091 (2014).

\section{Acknowledgements}

The authors acknowledge that this project has received funding from the European Union's Horizon 2020 research and innovation programme under grant agreement No. 785219 - GrapheneCore2. The authors also acknowledge that this project has received the financial support of the Bilateral Cooperation Agreement between Italy and China of the Italian Ministry of Foreign Affairs and of the International Cooperation (MAECI) and the National Natural Science Foundation of China (NSFC), in the framework of the project of major relevance 3-Dimensional Graphene: Applications in Catalysis, Photoacoustics and Plasmonics. Also, this work was supported by the Progetto FISR - C.N.R. "Tecnopolo di nanotecnologia e fotonica per la medicina di precisione" - CUP B83B17000010001.

\section{Author contributions}

F.D.N., I.V., L.D.T., F.R., F.S., R.A., M.R.L., A.S.N., X.F. and S.L. conceived the experiments. M.R.L. and A.S.N. prepared the graphene dispersion and F.R. and F.S. fabricated the samples and acquired the SEM images. F.D.N., I.V. and L.D.T. performed the wetting characterization of the samples and data analysis. All the authors discussed the experimental implementation, the results, and contributed in writing the paper.

\section{Competing interests}

The authors declare no competing interests.

\section{Additional information}

Supplementary information is available for this paper at https://doi.org/10.1038/s41598-020-58860-4.

Correspondence and requests for materials should be addressed to F.D.N.

Reprints and permissions information is available at www.nature.com/reprints.

Publisher's note Springer Nature remains neutral with regard to jurisdictional claims in published maps and institutional affiliations.

(c) (i) Open Access This article is licensed under a Creative Commons Attribution 4.0 International License, which permits use, sharing, adaptation, distribution and reproduction in any medium or format, as long as you give appropriate credit to the original author(s) and the source, provide a link to the Creative Commons license, and indicate if changes were made. The images or other third party material in this article are included in the article's Creative Commons license, unless indicated otherwise in a credit line to the material. If material is not included in the article's Creative Commons license and your intended use is not permitted by statutory regulation or exceeds the permitted use, you will need to obtain permission directly from the copyright holder. To view a copy of this license, visit http://creativecommons.org/licenses/by/4.0/.

(C) The Author(s) 2020 